\title{
Linear and ring polymers in confined geometries
}

\author{
Zoryana Usatenko ${ }^{1, a}$, Piotr Kuterba ${ }^{2}$, Hassan Chamati ${ }^{3}$, and Dirk Romeis ${ }^{4}$ \\ 1 Institute of Physics, Faculty of Physics, Mathematics and Computer Science, \\ Cracow University of Technology, 30-084 Cracow, Poland \\ 2 Faculty of Physics, Astronomy and Applied Computing, Jagiellonian University, \\ Cracow, Poland \\ 3 Institute of Solid State Physics, Bulgarian Academy of Sciences, 1784 Sofia, Bulgaria \\ ${ }^{4}$ Leibniz Institute for Polymer Research Dresden e.V., 01069 Dresden, Germany
}

Received 17 October 2016 / Received in final form 14 November 2016 Published online 5 April 2017

\begin{abstract}
A short overview of the theoretical and experimental works on the polymer-colloid mixtures is given. The behaviour of a dilute solution of linear and ring polymers in confined geometries like slit of two parallel walls or in the solution of mesoscopic colloidal particles of big size with different adsorbing or repelling properties in respect to polymers is discussed. Besides, we consider the massive field theory approach in fixed space dimensions $d=3$ for the investigation of the interaction between long flexible polymers and mesoscopic colloidal particles of big size and for the calculation of the correspondent depletion interaction potentials and the depletion forces between confining walls. The presented results indicate the interesting and nontrivial behavior of linear and ring polymers in confined geometries and give possibility better to understand the complexity of physical effects arising from confinement and chain topology which plays a significant role in the shaping of individual chromosomes and in the process of their segregation, especially in the case of elongated bacterial cells. The possibility of using linear and ring polymers for production of new types of nano- and micro-electromechanical devices is analyzed.
\end{abstract}

\section{Introduction}

Polymer-colloid mixtures have attracted a great deal of interest during the last few decades because of practical relevance, as well as for fundamental reasons [1-5]. In industrial products, such as paint, food, composite materials, derived from mixing together polymers and colloids phase stability is a question of vital importance. Thus it is very important to determine the phase behavior of such mixtures, taking into account the microscopic interaction between colloidal particles in polymer solutions. During the investigation of the microscopic interactions in polymer-colloid mixtures we can distinguish two cases which usually lead to qualitatively different effects.

\footnotetext{
${ }^{a}$ e-mail: zusatenko@pk.edu.pl
} 
The first case is connected with the investigation of such polymer-colloid mixtures where polymers are adsorbed on the colloidal surfaces and protect colloids from flocculation. In this case colloidal surfaces are maintained at large enough separations because adsorbed polymers resist the ability to approach the other surfaces and as a result protect against arising attraction due to the depletion effect or London-van der Waals force and it leads to stabilization of the colloidal suspension [6,7]. The second case is connected with investigation of a mixture of colloidal particles and nonadsorbing polymers which leads to arising of the so called depletion effect [8] when polymers are expelled from the region between two colloidal particles or between colloidal particle and a wall due to entropic reasons. It leads to an unbalanced pressure on the outside which pushes colloidal particle to a wall or two colloidal particles towards each other. In the latter case a key role is played by the depletion potential giving rise to, among others, the depletion force between colloidal particles or between a colloidal particle and a wall, respectively. The force's magnitude is affected by the concentration of polymer solutions and the effective size of polymer coils, as well as the size and the shape of the confining colloidal particles and width of their separation. The improvement of the experimental technique allowed even to measure with high accuracy the depletion force which arises between a single colloidal particle and a wall [8-10]. Furthermore the force enters into a complex force balance with other intercolloidal interactions, such as DLVO, hydration, hydrophobic interaction $[4,5,11]$. Therefore it is sometimes challenging to separate and quantify its contribution. An idealized physical model for these systems was proposed some time ago by Asakura and Oosawa $[12,13]$ which was based on a mixture of hard spheres with different sizes to describe the colloids dispersed in a solution of free nonadsorbing polymers playing the role of depletants. The problem is that the common Asakura-Oosawa approximation modeling the polymer coils as hard spheres turns out to fail completely for small particles and fails by about $10 \%$ for large particles because long polymers actually are a flexible objects and, in general, cannot be modeled as hard spheres. In accordance with it the approaches which take into account the polymer chain flexibility are more effective. In the case of strongly overlapping polymer chains in semidilute solutions the chain flexibility was taken into account in the framework of the phenomenological scaling theory [14-16] and via the self-consistent field theory $[17,18]$. In the case of sufficiently dilute polymer solutions, different polymers do not overlap and the behaviour of such solution can be modeled by a random walk (RW) of a single polymer chain for ideal polymer in $\Theta$ - solvent $[15,16,19,20]$ or self-avoiding walk (SAW) for the case of real polymer with the excluded volume interaction (EVI) in a good solvent at the temperatures above the $\Theta$ - temperature $[15,16]$. The situation when the solvent temperature is below the $\Theta$ - temperature corresponds to poor solvent where polymer coils tend to collapse $[21,22]$. A remarkable progress in the investigation of the depletion interaction potentials and the depletion forces which arise in the case of immersing the polymer solution of linear ideal and real polymer chains with the EVI inside the slit geometry of two parallel walls was achieved in [23,24] via the dimensionally regularised continuum version of the field theory with minimal subtraction of poles in the $\epsilon$ - expansion for the case of two repulsive walls and in one of our papers [25] via the massive field theory approach for the case of two repulsive walls, two inert walls and for the mixed situation of one repulsive and the other one inert wall. It should be mentioned, that our results obtained in [25] showed that the value of the depletion force in the case of one repulsive and the other one inert wall is smaller than in the case of two repulsive walls. Besides, in [25] we obtained that the depletion force in the case of two inert walls (the walls where the adsorption threshold takes place) becomes repulsive for the case of real polymers with the EVI in a good solvent. This result is very important from practical reasons, because it means that in such systems we observe reduction of the static friction and as a result such systems can 
be used for producing of new types of nano- and micro-mechanical devices. Besides, the calculation of the depletion force was performed by numerical methods [26-28] using the model of RW for ideal polymer at $\Theta$ - solvent and SAW for a real polymer with the EVI in a good solvent confirmed inside the slit of two repulsive walls. The validity of the universal density-force relation proposed by Joanny, Leibler and de Gennes [14] for the different cases of a single polymer with one end (or both ends) fixed in the half space bounded by the wall, a single chain trapped in a slit geometry of two parallel walls, for the case of a dilute and semi-dilute solution of free polymers in a half space and for the case of polymer in a half space containing a mesoscopic colloidal particle of arbitrary shape was discussed initially in [23]. The above mentioned universal density-force relation was verified by simulation using an off-lattice beadspring model of a polymer chain trapped between two parallel repulsive walls $[26,27]$ and by the lattice Monte Carlo (MC) algorithm on a regular cubic lattice in three dimensions [28]. In a series of our papers [29,30] the universal density-force relation is analyzed by analogy as it was proposed by Eisenriegler [23] and the corresponding universal amplitude ratio was obtained in the framework of the massive field theory approach directly in fixed space dimensions $d=3$. It allowed us to obtain in $[29,30]$ the monomer density profiles of linear ideal and real polymers with the EVI in a good solvent immersed in a slit geometry of two parallel repulsive walls, one repulsive and the other one inert wall. Besides, the monomer density profiles of a dilute polymer solution confined in a semi-infinite space containing the mesoscopic spherical colloidal particle of big radius were calculated. It should be mentioned, that the interaction of long flexible nonadsorbing linear polymers with mesoscopic colloidal particles of big and small size and different shape was the subject a series of papers $[31,32]$. The obtained results for long flexible linear polymer chains indicate that focusing on such systems leads to universal results which are independent of microscopic details and are free of nonuniversal model parameters and depend only on shapes of particles and ratios of three characteristic lengths of the system such as the radius of the particle, the polymer size, the distance between the particle and the wall or between two particles, respectively.

The other very interesting question is investigation of the influence of the polymer chain topology on the depletion interaction potentials and depletion forces. In this respect it is necessary to mention polymers with ring topology. As it was shown in a series of the atomic force spectroscopy (AFM) experiments [33,34] biopolymers such as DNA very often present ring topology. Such situation takes place, for example, in the case of Escherichia coli (E.coli) bacteria with a chromosome which is not a linear polymer, but has a ring topology [35]. The biopolymers of DNA of some viruses such as bacteriophages $\lambda$ that infect bacteria oscillate between linear and ring topology $[36,37]$. The linear form of DNA is encountered in mature viruses, however inside of the host cell DNA of phages adopts a ring topology [38]. Besides, the physical effects arising from confinement and chain topology play a significant role in the shaping of individual chromosomes and in the process of their segregation, especially in the case of elongated bacterial cells [39].

Ring polymers with specified knot type were chemically synthesized a long time ago [40]. Ring topology has an influence on the statistical mechanical properties of polymers, for example, on scaling properties [41,42] and shape $[34,43,44]$ because it restrains the accessible phase space. Looking back into the history of the investigation of the statistical mechanical properties of ring polymers we should mention that an interesting point which was confirmed by numerical studies in [45] is that longer ring polymers are usually knotted with higher frequency and complexity. In [46] it was established, that ring polymers with more complex knots are more compact and have a smaller radius of gyration and this decreases their ability to spread out under confinement. A series of papers have been devoted to the investigation of 
ring polymers compressed or squeezed by a force in a slab [47-49]. For example, the results of MC simulations performed in [47] suggest that the knotted ring polymers will exert higher entropic forces on the walls of the confining slit than unknotted or linear polymers. In [47] it was stated that the knotted ring polymers expanded as the width of the slit increased in contrast to the behaviour of unknotted (or linear) polymers whose size showed a plateau after a certain width of slit was reached. The entropic force exerted on the walls arising from confinement to a slit of a knotted ring polymer was calculated using a bead-spring model by Matthews et al. [37]. It was found [37] that in the case of a narrow slit more complex knot types in a ring polymer exert higher forces on the confining walls of the slit in comparison to unknotted polymer chains of the same length, and for the relatively wide slits the opposite situation takes place. As it was shown in [49], confining ring polymer to a slab results in the loss of configurational entropy and leads to the arising of a repulsive force which depends on the entanglements between the two walls of the confining slab. It should be mentioned, that in [49] the profiles of critical forces which are necessary to apply in order to overcome this entropically induced repulsion were obtained in the framework of a new numerical approach which was the implementation of the generalized atmospheric sampling (GAS) algorithm for lattice knots proposed by Rensburg and Rechnitzer in [50]. Recently advanced MC simulation techniques [51] have been used in order to study the effect of nanoslit confinement on topological properties of circular model DNA, which was modeled as a semiflexible polymer chain. They have shown that the knotting probability has strong slit width dependence. Besides, the investigation of the influence of topological constraints on the free energy and metric properties of an ideal ring polymers without excluded volume effects or attractive interactions confined in a narrow slit were performed using off-lattice MC simulations in [52]. It was found [52] that the scaling behavior of the confinement free energy of a freely jointed ring and an unknotted ring polymer is the same in a narrow slit region.

In a series of recent papers based on MC simulations [53-55] the occurrence and behaviour of knots in polymers were studied extensively. Such, for example, in [53] numerical simulations were used in order to show that the effective stiffening of DNA by the nematic arrangement promotes formation of torus knots in phage capsids. In [54] a mechanism in which two knots can pass through each other and swap positions along the DNA strand were suggested and explained. Using a combination of the multicanonical MC algorithm and the replica - exchange method in [55] were performed investigation of the influence of bending stiffness on the conformational phases of a bead - stick polymer and obtained the corresponding pseudophase diagram for flexible, semiflexibe and stiff polymers. Besides, in [55] was found the thermodynamically stable knots and unusual transitions into these knotted phases.

The question of influence of the polymer chain topology on the depletion interaction potentials and the depletion forces was investigated in a series of our papers [56-58], where we performed the investigation of a dilute solution of ring polymers immersed in a confined geometry like slit of two parallel walls with different boundary conditions and in a solution of mesoscopic colloidal particles of one sort or two sorts which can be both attractive, both repulsive for polymers or discuss the mixed case when one sort of mesoscopic colloidal particles is attractive for ring polymers and the other one is repulsive. It should be mentioned, that our investigation and calculation of the respective depletion interaction potentials and the depletion forces for linear and ring polymers with the EVI in a good solvent immersed in confined geometries is based on one of the powerful analytical method: the massive field theory approach in fixed space dimensions $d=3$ (see $[25,29,30,59])$ up to one-loop order, which demonstrates quite good agreement with the results of numerical calculations and experimental data. 


\section{The thermodynamic description and the model}

In a series of our papers (see $[25,29,30,56-58]$ ) the investigations of a dilute solution of linear or ring polymers confined in a slit geometry of two parallel walls and in a solution of mesoscopic spherical colloidal particles of one sort or two different sorts were performed. We assumed that the solution of polymers is sufficiently dilute so that the interchain interactions and the overlapping between different polymers can be neglected and it is sufficient to consider the behaviour of a single polymer chain. Besides, as it was confirmed by multicanonical simulations recently [60], polymers in a dilute solution confined in a spherical cavity can be considered isolated for all temperatures larger than the aggregation temperature, which is a function of the density. As it is known [15,16,61,62], the behaviour of long flexible polymers is determinated by entropy and characterized by detail independence, universality and scaling. The properties of universality and scaling also took place for critical systems with surfaces $[64,65]$ and can be applied for the case of polymer solution in confined geometries $[24,25,56,57,62]$.

It should be mentioned that in a series of our papers $[25,56-58]$ we followed the thermodynamic description of the problem as it was proposed in [24] and performed at the beginning the calculations in the framework of the grand canonical ensemble where the chemical potential $\mu$ is fixed and later obtained the results for the canonical ensemble via the Legendre transform. We performed the investigation of a dilute solution of linear or ring polymers immersed in the slit geometry of two parallel walls and allowed for the exchange of polymer coils between the slit and the reservoir. In such a situation the polymer solution in the slit is in equilibrium contact with an equivalent solution in the reservoir outside the slit. As it was shown in [24], the free energy of the interaction between the walls in such a grand canonical ensemble is defined as the difference of the free energy of an ensemble where the separation of the walls is fixed at a finite distance $L$ and where the walls are separated infinitely far from each other:

$\delta F=-k_{B} T \tilde{N} \ln \left(\frac{\mathcal{Z}_{\|}(L)}{\mathcal{Z}_{\|}(L \rightarrow \infty)}\right)=-k_{B} T \tilde{N}\left\{\ln \left(\frac{\mathcal{Z}_{\|}(L)}{\mathcal{Z}}\right)-\ln \left(\frac{\mathcal{Z}_{\|}(L \rightarrow \infty)}{\mathcal{Z}}\right)\right\}$,

where $\tilde{N}$ is the total amount of polymer coils in the solution and $T$ is the temperature. The $\mathcal{Z}_{\|}(L)$ value is the partition function of one polymer (linear or ring) located in a volume $V$ containing two walls at a distance $L$. It should be mentioned that we normalized the partition functions $\mathcal{Z}_{\|}(L)$ and $\mathcal{Z}_{\|}(L \rightarrow \infty)$ on the function $\mathcal{Z}=V \hat{\mathcal{Z}}_{b}$, where $\hat{\mathcal{Z}}_{b}=\mathcal{I} \mathcal{L}_{\mu_{0}^{2}->\frac{R_{x}^{2}}{2}}\left[\frac{1}{\mu_{0}^{2}}\right]$ for linear polymers and $\hat{\mathcal{Z}}_{b}=\mathcal{I} \mathcal{L}_{\mu_{0}^{2}->\frac{R_{x}^{2}}{2}}\left[\frac{1}{2 \mu_{0}}\right]$ for ring polymers, respectively.

Following the thermodynamic description proposed in $[24,25,56,57]$ the corresponding reduced free energy of interaction $\delta f$ per unit area $A=1$ for the case of linear or ring polymers confined in the slit geometry of two parallel walls after performing Fourier transform in the direction parallel to the surfaces and integration over $d^{d-1} r$ can be obtained:

$$
\delta f=\frac{\delta F}{n_{p} k_{B} T} .
$$

Here $n_{p}=\tilde{N} / V$ is the polymer density in the bulk solution. The reduced free energy of interaction $\delta f$, according to equation (2), is a function of the dimension of length and dividing it by another relevant length scale, for example, the size of polymer in bulk, e.g. $R_{x}$ yields a universal dimensionless scaling function for the depletion interaction potential:

$$
\Theta(y)=\frac{\delta f}{R_{x}}
$$


where $y=L / R_{x}$ is a dimensionless scaling variable. The resulting scaling function for the depletion force between two walls induced by the polymer solution is denoted as:

$$
\Gamma(y)=-\frac{d(\delta f)}{d L}=-\frac{d \Theta(y)}{d y} .
$$

As it is known [62], the force exerted on the surfaces of a confining slit by polymer is equal to the depletion force with opposite sign: $K(y)=\frac{d(\delta f)}{d L}$.

In general the behavior of a single ideal (Gaussian) polymer at $\theta$-solvent can be described by a model of random walk (RW) and the behavior of real polymer with the EVI for temperatures above the $\theta$-point by a model of self-avoiding walk (SAW). Usually in the case when the EVI between monomers becomes relevant the polymer coils are less compact than in the case of ideal chains. As it is known, taking into account the polymer-magnet analogy developed by de Gennes $[15,16]$, their scaling properties in the limit of an infinite number of steps $N$ may be derived from a formal $n \rightarrow 0$ limit of the field theoretical $\phi^{4} O(n)$ - vector model at its critical point. In this case the value $1 / N$ plays the role of a critical parameter analogous to the reduced critical temperature in magnetic systems. In the case when the polymer solution is in contact with solid substrates, the monomers interact with the surfaces. In a series of papers [66-68] the extensive multicanonical MC computer simulations were used in order to study the conformational behaviour of flexible polymers near an attractive surfaces and the corresponding complete solubility-temperature phase diagram was constructed and discussed. The proposed in [24,25,56-58] calculations were performed for the case when the surfaces were impenetrable. It means that the corresponding potential $U(z)$ of the interaction between the monomers of a polymer chain and a wall tends to infinity $U(z) \rightarrow \infty$ when the distance between a wall and polymer chain is less than monomer size $l$. The deviation from the adsorption threshold $\left(c \propto\left(T-T_{a}\right) / T_{a}\right)$ (where $T_{a}$ is adsorption temperature) changes sign at the transition between the adsorbed (the so-called normal transition, $c<0$ ) and the nonadsorbed state (ordinary transition, $c>0)[62,65]$ and it plays the role of a second critical parameter. The value $c$ corresponds to the adsorption energy divided by $k_{B} T$ (or the surface enhancement in field theoretical treatment). The adsorption threshold for long-flexible polymers takes place, where $1 / N \rightarrow 0$ and $c \rightarrow 0$. As it was shown in $[24,25,56,57]$, in the case when polymer solution is confined in a slit geometry of two parallel walls the properties of the system depend on the ratio $L / \xi_{R}$, where $L$ is the the distance between two walls and $\xi_{R}=\sqrt{\left\langle R^{2}\right\rangle} \sim N^{\nu}$ is the average end-to-end distance.

As was mentioned by de Gennes $[15,16]$, the partition function $Z\left(\mathbf{x}, \mathbf{x}^{\prime}\right)$ of a single polymer chain with two ends fixed at $\mathbf{x}$ and $\mathbf{x}^{\prime}$ is connected with the two-point correlation function $G^{(2)}\left(\mathbf{x}, \mathbf{x}^{\prime}\right)=\left\langle\phi(\mathbf{x}) \phi\left(\mathbf{x}^{\prime}\right)\right\rangle$ in $\phi^{4} O(n)$ - vector model for $n$-vector field $\phi(\mathbf{x})$ with the components $\phi_{i}(x), i=1, \ldots, n$ (and $\mathbf{x}=(\mathbf{r}, z)$ ) via the inverse Laplace transform $\mu_{0}^{2} \rightarrow L_{0}: Z\left(\mathbf{x}, \mathbf{x}^{\prime} ; N, v_{0}\right)=\mathcal{I} \mathcal{L}_{\mu_{0}^{2} \rightarrow L_{0}}\left(\left.\left\langle\phi(\mathbf{x}) \phi\left(\mathbf{x}^{\prime}\right)\right\rangle\right|_{n \rightarrow 0}\right)$ in the limit, where the number $n$ of components tends to zero. The conjugate Laplace variable $L_{0}$ has the dimension of length squared and is proportional to the total number of monomers $N$ of the polymer chain: $L_{0} \sim l^{2} N$.

The effective Ginzburg-Landau-Wilson Hamiltonian describing the system of a dilute polymer solution confined in semi-infinite $(i=1)$ or confined geometry of two parallel walls $(i=1,2)$ is $[65]$ :

$$
\mathcal{H}\left[\phi, \mu_{0}\right]=\int d^{d} x\left\{\frac{1}{2}(\nabla \phi)^{2}+\frac{\mu_{0}^{2}}{2} \phi^{2}+\frac{v_{0}}{4 !}\left(\phi^{2}\right)^{2}\right\}+\sum_{i=1}^{2} \frac{c_{i_{0}}}{2} \int d^{d-1} r \phi^{2},
$$

where the conjugate chemical potential $\mu_{0}$ is the "bare mass" in field - theoretical treatment, $v_{0}$ is the "bare coupling constant" which characterizes the strength of the 
EVI. In the case of a slit geometry the walls are located at the distance $L$ one from another in $z$-direction in such way that the surface of the bottom wall is located at $z=0$ and the surface of the upper wall is located at $z=L$. Each of the two system surfaces is characterized by a certain surface enhancement constant $c_{i_{0}}$, where $i=1,2$. In the case when the ends of polymer chain $\mathbf{x}=(\mathbf{r}, z)$ and $\mathbf{x}^{\prime}=\left(\mathbf{r}^{\prime}, z^{\prime}\right)$ in partition function coincide, such partition function corresponds to the partition function of a phantom ring polymer, i.e. a ring polymer where we perform the summation over all possible knot structures [56-58].

The interaction between the polymer chain and the walls is implemented by the different boundary conditions. In a series of our papers we performed investigation for different boundary conditions. Such, for example, we performed investigation for the case of two repulsive walls, where the segment partition function and thus the partition function for the whole polymer tends to zero as any segment approaches the surface of the walls. This case corresponds to the Dirichlet-Dirichlet boundary conditions (D-D b.c.) (see also $[24,25,65])$ :

$$
\phi(\mathbf{r}, 0)=\phi(\mathbf{r}, L)=0 \quad \text { or } \quad c_{1} \rightarrow+\infty, \quad c_{2} \rightarrow+\infty .
$$

Besides, we performed calculations for the case of two inert walls, which corresponds to the situation of Neumann-Neumann boundary conditions (N-N b.c.) (see also $[25,65]$ ):

$$
\left.\frac{\partial \phi(\mathbf{r}, z)}{\partial z}\right|_{z=0}=\left.\frac{\partial \phi(\mathbf{r}, z)}{\partial z}\right|_{z=L}=0 \quad \text { or } \quad c_{1}=0, \quad c_{2}=0
$$

and for the mixed case of one repulsive and the other one inert wall, where DirichletNeumann boundary conditions(D-N b.c.) takes place:

$$
\phi(\mathbf{r}, 0)=0,\left.\quad \frac{\partial \phi(\mathbf{r}, z)}{\partial z}\right|_{z=L}=0 \quad \text { or } \quad c_{1} \rightarrow+\infty, \quad c_{2}=0 .
$$

It should be mentioned that the term "inert" in this contents means the surface where the adsorption threshold takes place. In fact the performed calculations are valid for the case of wide slit limit $y \geq 1$ (where $y=\frac{L}{R_{x}}$ ), but they are not suitable to describe the case of dimensional crossover from $d$ to $d-1$ dimensional system which arises for $y \ll 1$. The $d-1$ dimensional system is characterized by another critical temperature and a new critical fixed point, as it takes place, for example, in magnetic or liquid thin films. Nevertheless, some assumptions allowed us to describe the region of narrow slit, as it was proposed in one of our papers (see Ref. [25]). In the case of infinitely large wall separations, the slit system decomposes into two half-space systems.

It should be mentioned that the most common parameter in polymer physics used for the signification of polymers size which is observable in experiments is the radius of gyration $R_{g}$ (see Refs. [61,62,69]): $R_{g}^{2}=\chi_{d}^{2} \frac{R_{x}^{2}}{2}$, where $\chi_{d}$ is a universal numerical prefactor depending on the dimension $d$ of the system and $R_{x}^{2}=\frac{l^{2} N^{2 \nu}}{3}$ (where $\nu$ is 0.5 for ideal polymers and 0.588 for real polymers with the EVI). For ideal polymers takes place the relation: $\chi_{d}^{2}=\frac{d}{3}$ and for real polymers at $d=3$ the following takes place $[61]: \chi_{3}^{2}=0.958$.

\section{Dilute solution of ideal polymers in a slit geometry}

Let's consider at the beginning the case of a dilute solution of ideal polymers in a slit geometry of two parallel walls. As it was mentioned above we are interested in 
discussion of two cases: $(a)$ ideal linear polymer and $(b)$ phantom ideal ring polymer under $\Theta$-solvent condition trapped in a slit geometry of two parallel walls situated at a distance $L$ one from another with different boundary conditions (see Eqs. (6)-(8)).

(a) Ideal linear polymer in a slit geometry of two parallel walls. In [25] for the case of ideal linear polymer in a slit geometry of two parallel walls with D-D b.c. (6) we obtained that if both $c_{1}$ and $c_{2}$ are positive, the depletion interaction potential $\Theta_{D D}(y)$ is negative and hence the walls attract each other due to the depletion zones near repulsive walls. It should be mentioned, that the obtained results for ideal linear polymer in a slit geometry of two repulsive walls are in agreement with the previous theoretical results obtained in reference [24]. For the dimensionless scaling function of the depletion interaction potential in the case of two inert walls, which corresponds to N-N b.c. (7) in [25] we obtained $\Theta_{N N}(y)=0$. It corresponds to the fact that ideal chains do not loose free energy inside the slit in comparison with free chains in unrestricted space. The entropy loss is fully regained by surface interactions provided by two walls. In the case of one repulsive and the other one inert wall, which corresponds to D-N b.c. (8) we obtained (see [25]) that $\Theta_{D D}(2 y) \approx 2 \Theta_{D N}(y)$. The obtained result is intuitively clear, because the depletion zone is formed only near the lower wall, i.e. near the wall with Dirichlet b.c. The upper wall with Neumann b.c. does not contribute at all to the induced depletion interaction. It should be mentioned that in [25] for the case of ideal linear polymer chain confined in a slit geometry of two parallel walls with different boundary conditions the exact results for the dimensionless scaling functions of the depletion interaction potential and the depletion force were obtained. Besides, in [25] the asymptotic region of wide slit with $y \geq 1$ and narrow slit with $y \ll 1$ were discussed. In the case of two repulsive walls and for the mixed case of one repulsive and the other one inert wall in the wide slit region with $y \geq 1$ the following results for the dimensionless scaling functions of the depletion force in [25] were obtained, respectively:

$$
\Gamma_{D D}(y) \approx-4 \operatorname{erfc}\left(\frac{y}{\sqrt{2}}\right)+8 \operatorname{erfc}(\sqrt{2} y), \quad \Gamma_{D N}(y) \approx-4 \operatorname{erfc}(\sqrt{2} y) .
$$

The results obtained in [25] for the dimensionless scaling functions of the depletion force are presented in Figure 1a-1c by black lines with black squares, respectively.

In the case of narrow slit with $y \ll 1$ the asymptotic solution for the depletion force in both the above mentioned cases simply becomes $\Gamma_{D D(D N), n a r r}(y) \approx-1$ (see [25]). These results can be understood phenomenologically. In our units the quantities $\Theta$ and $\Gamma$ were normalized to the overall polymer density $n_{p}$. So, the above results simply indicate that the force is entirely induced by free chains surrounding the slit, or, in other words, by the full bulk osmotic pressure from the outside of the slit. No chain has remained in the slit. It is reasonable in the case of repulsive walls in the limit of narrow slits. The above mentioned arguments were used in order to obtain the leading contributions to the depletion effect as $y \rightarrow 0$. We can state that in the case of very narrow slits polymers would pay a very high entropy to stay in the slit or even enter it. It is due to the fact that the phase space containing all possible conformations is essentially reduced by the squeezing confinement to the size $\frac{d-1}{d}$ times its original size (for an unconfined chain). Therefore, as it was shown in [25], the ratio of partition function of polymer chain in the slit and the free chain partition function vanishes strongly as $y \rightarrow 0$. The advantage of the proposed procedure is that no expansion is necessary in this case of narrow slit region and it should be equally valid for polymers with the EVI.

(b) Phantom ideal ring polymer in a slit geometry of two parallel walls. In $[56,57]$ the dimensionless scaling functions of the depletion interaction potential $\Theta^{R}(y)$ and the depletion force $\Gamma^{R}(y)$ between two repulsive, two inert and for the mixed case of one repulsive and the other one inert wall induced by the dilute solution 
of phantom ideal ring polymers in the wide slit region $y \geq 1$ were obtained. The corresponding dimensionless scaling functions of the depletion force for D-D (N-N) b.c. and D-N b.c. are:

$$
\Gamma_{D D}^{R}(y)=2 e^{-2 y^{2}}-8 y^{2} e^{-2 y^{2}}, \quad \Gamma_{D N}^{R}(y)=-2 e^{-2 y^{2}}+8 y^{2} e^{-2 y^{2}} .
$$

We obtained that phantom ideal ring polymers due to the complexity of chain topology and from entropical reasons prefer to escape from the space not only between two repulsive walls but also in the case of two inert walls. As a result, the attractive depletion force arises between the walls. As it is possible to see from equation (10), the dimensionless scaling function of the depletion force in the case of mixed boundary conditions becomes repulsive in the region $y \geq 1$.

Taking into account the above mentioned arguments (see section $3(a)$ ) in the case of narrow slit of two repulsive walls with $y \rightarrow 0$ the asymptotic solution for the scaling function of the depletion force simply becomes: $\Gamma_{D D, n a r r}^{R}(y) \approx-1$ (see $\left.[56,57]\right)$. We obtained that the scaling behaviour of the depletion force is the same for linear and ring polymers in a narrow slit region of two parallel repulsive walls. The obtained results for the scaling functions of the depletion force in the case of linear and ring polymers in a narrow slit are in agreement with the results obtained in [52].

\section{Dilute solution of linear and ring polymers with the EVI in a slit geometry}

As a next step let's consider a dilute solution of linear and ring polymers with the EVI in a good solvent immersed in a slit geometry of two parallel walls. As it is known [15,16], in a good solvent the effects of the EVI between monomers play a crucial role so that the polymer coils occupy large space and are less compact than in the case of ideal polymers. The influence of the EVI on the depletion functions and corresponding partition functions can be obtained using perturbation treatment in the framework of the massive field theory approach in fixed space dimensions $d=3$ up to one-loop order approximation in the presence of confining slit geometry of two parallel walls. In order to make the theory ultraviolet (UV) finite in RG sense directly in $d=3$ space dimensions we performed the standard mass renormalization $\mu_{0}^{2}=\mu^{2}-\delta \mu_{0}^{2}$ and the coupling constant renormalization $v_{0}=\mu v$ of the corresponding correlation functions by analogy as it was proposed by Parisi [70]. Besides, in order to remove the UV singularities of the correspondent correlation functions on the surface the surface enhancement renormalization $c_{j_{0}}=c_{j}+\delta c_{j}$ were performed (see $[25,56$, 57]) by analogy as it took place in the case of semi-infinite geometry [59]. It should be mentioned that the calculations were performed at the corresponding stable fixed point $\tilde{v}^{*}=1$ obtained from ressummed beta functions of the underlying bulk field theory in the framework of one-loop approximation scheme. The obtained in [25] results for the dimensionless scaling functions of the depletion force $\Gamma(y)$ between two repulsive walls, two inert walls and for one repulsive and the other one inert wall in the case of a dilute solution of linear polymers with the EVI in a good solvent are presented in Figure 1a-1c by red lines with red points, respectively. The respective results for the dimensionless scaling function of the depletion force between two repulsive walls in the case of a dilute solution of ring polymers with the EVI are presented in Table 1. 

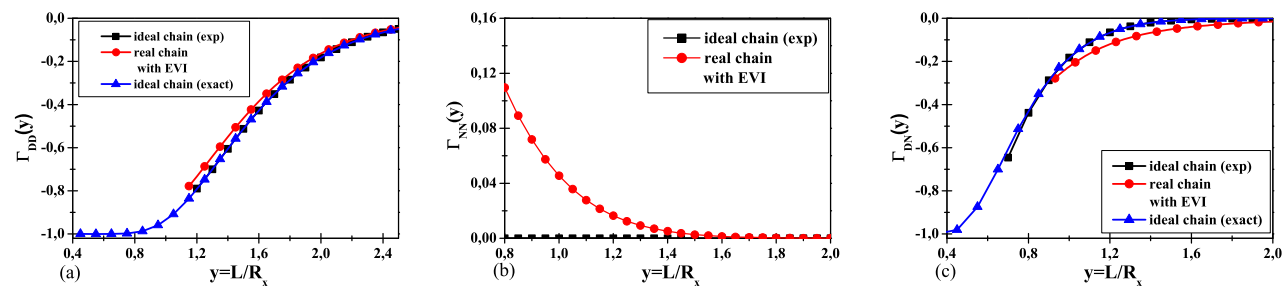

Fig. 1. The dimensionless scaling functions of $\Gamma(y)$ for a dilute solution of linear polymers immersed in a slit geometry with different boundary conditions (see [25]): (a) D-D b.c.; (b) N-N b.c.; (c) D-N b.c.

Table 1. The dimensionless scaling functions of $\Gamma_{D D}^{R}$ between two repulsive walls as function of $y=L / R_{x}$ in the case of a dilute solution of ring polymers with the EVI.

\begin{tabular}{llllll}
\hline$y$ & 1.3 & 1.5 & 1.7 & 1.9 & 2.1 \\
\hline ideal ring & -0.392 & -0.178 & -0.065 & -0.020 & -0.005 \\
ring with EVI & -0.391 & -0.175 & -0.064 & -0.019 & -0.005 \\
\hline
\end{tabular}

\section{Linear and ring polymers in a solution of mesoscopic spherical colloidal particles}

Another interesting situation arises when we have a dilute solution of linear or ring polymers immersed in the solution of mesoscopic colloidal particles. The interaction of long flexible nonadsorbing linear polymers with mesoscopic colloidal particles of big or small size with different shape was the subject of investigation in a series of papers $[31,32]$. The performed in $[31,32]$ investigations for long flexible linear polymers indicate that focusing on such systems leads to universal results which are independent of microscopic details and are free of nonuniversal model parameters and depend only on shapes of particles and ratios of three characteristic lengths of the system such as the radius of the particle, the polymer size, the distance between the particle and the wall or between two particles, respectively. In our papers $[25,56,58]$ we focused our attention on the investigation of the depletion interaction and the depletion force for two cases: (1) between a big spherical colloidal particle of radius $R$ and the wall; (2) between two colloidal particles of big sizes with different radii $R_{1}$ and $R_{2}$ and different adsorbing or repelling properties with respect to polymers in the solution. The interaction of a dilute solution of linear or ring polymers with particles and walls is implemented by the corresponding boundary condition. The difference between the forces with and without the particle (or particles) yields the depletion interaction of the particle with the wall (or between two particles). Taking into account the Derjaguin approximation [71], which describes the sphere of the big colloidal particle of the radius $R$ (with $R \gg L$ and $R \gg R_{x}$ ) by a superposition of immersed plates (see Figure 2a) with local distance $h(\rho)=a+R-\sqrt{R^{2}-\rho^{2}}$ from the wall, where $a-$ is the nearest distance from the particle to the wall and $\rho-$ is the width of the fringe itself we performed calculations of the depletion interaction potential $\Phi(\tilde{y}) / n_{p} k_{B} T$ and the depletion force $\frac{-d\left(\Phi(\tilde{y}) / n_{p} k_{B} T\right)}{d \tilde{y}}$ between the colloidal particle and the wall in a dilute solution of ring polymers. It should be mentioned that we also used some modifications of the Derjaguin approximation for the calculation of the depletion interaction potential and the depletion force in the case of two big spherical colloidal particles with different radii $R_{1} \neq R_{2}$ when $R_{i} \gg L$ and $R_{i} \gg R_{x}, i=1,2$. In this case we took into account that the distance $h(\rho)$ is equal to: $h(\rho)=a+R_{1}-\sqrt{R_{1}^{2}-\rho^{2}}+R_{2}-\sqrt{R_{2}^{2}-\rho^{2}}$, where $a-$ in this case is the 

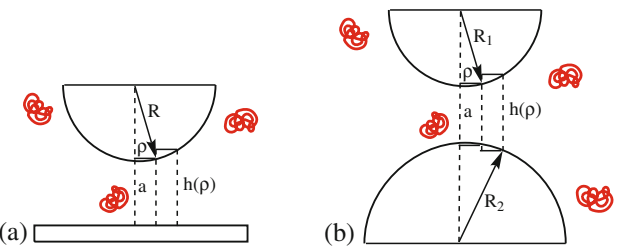

Fig. 2. The Derjaguin approximation (see [71]).
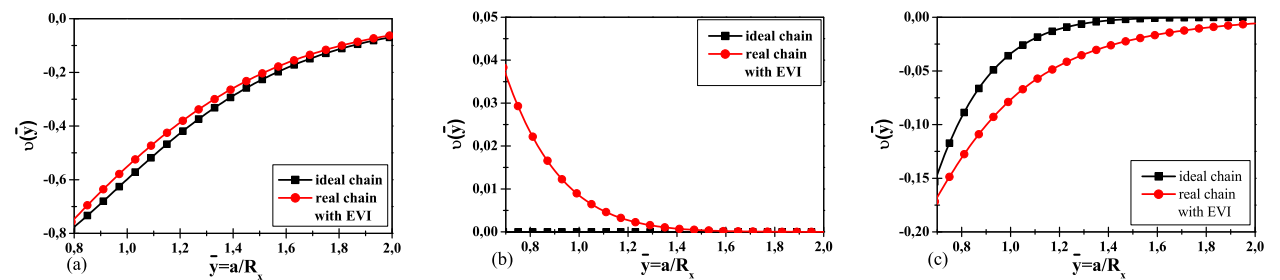

Fig. 3. The dimensionless scaling functions of $\vartheta(\tilde{y})$ for a dilute solution of linear polymers immersed between the big spherical colloidal particle and the wall (or between two big spherical colloidal particles) for the case of (a) D-D b.c.; (b) N-N b.c.; (c) D-N b.c.

nearest distance from the particle to other particle (see Fig. $2 \mathrm{~b}$ ). The depletion interaction potential $\Phi(\tilde{y}) / n_{p} k_{B} T$ is equal to: $2 \pi \tilde{R} R_{x}^{2} \int_{\tilde{y}}^{\infty} d y \Theta(y)$ with $\tilde{y}=a / R_{x}$ where $\tilde{R}=R$ for the case of the big spherical colloidal particle of radius $R$ near the wall and $\tilde{R}=R_{1} R_{2} /\left(R_{1}+R_{2}\right)$ for the case of two big spherical colloidal particles with different radii $R_{1} \neq R_{2}$. Based on the results for the depletion interaction potential $\Phi(\tilde{y}) / n_{p} k_{B} T$ we can calculate the depletion force for certain b.c.'s. The depletion force between the big spherical colloidal particle and the wall (or between two big spherical colloidal particles) has the following form $[56,58]$ :

$$
-\frac{d}{d \tilde{y}} \frac{\Phi^{i d}(\tilde{y})}{n_{p} k_{B} T}=2 \pi R_{x}^{2} \tilde{R} \vartheta^{i d}(\tilde{y})
$$

where $\vartheta^{i d}(\tilde{y})$ is equal to: $\pm 2 \tilde{y} e^{ \pm 2 \tilde{y}^{2}}$ for ideal ring polymers, where the sign "-" corresponds to D-D b.c. or N-N b.c. and the sign "+" to the case of D-N b.c. The results for the corresponding scaling functions $\vartheta^{i d}(\tilde{y})$ for the case of a dilute solution of ideal linear polymer chains are presented in Figure 3a-3c by black lines with black squares, respectively. It should be mentioned that in a similar way we performed calculations for the depletion force between the big colloidal particle and the wall (or between two big spherical colloidal particles of different radii) induced by a dilute solution of linear and ring polymers with the EVI in a good solvent.

The obtained results for the corresponding dimensionless scaling functions $\vartheta^{E V I}(\tilde{y})$ of the depletion force in the case of linear polymer chains with the EVI immersed between the big spherical colloidal particle and the wall (or between two big spherical colloidal particles) with different boundary conditions are presented in Figure $3 \mathrm{a}-3 \mathrm{c}$ by red lines with red points, respectively. Besides, the corresponding results for the dimensionless scaling function of the depletion force in the case of ring polymer with the EVI immersed between the big spherical colloidal particle and the wall (or between two big spherical colloidal particles) with D-D b.c. are presented in Table 2. 
Table 2. The dimensionless scaling function of $\vartheta^{R}(\tilde{y})$ for a dilute solution of ring polymers immersed between the big spherical colloidal particle and the wall (or between two big spherical colloidal particles) in the case of D-D b.c. as function of $\tilde{y}=a / R_{x}$.

\begin{tabular}{llllll}
\hline$y$ & 1.3 & 1.5 & 1.7 & 1.9 & 2.1 \\
\hline ideal ring & -0.0885 & -0.0333 & -0.01050 & -0.00278 & -0.0006 \\
ring with EVI & -0.0873 & -0.0326 & -0.01025 & -0.00271 & -0.0006 \\
\hline
\end{tabular}
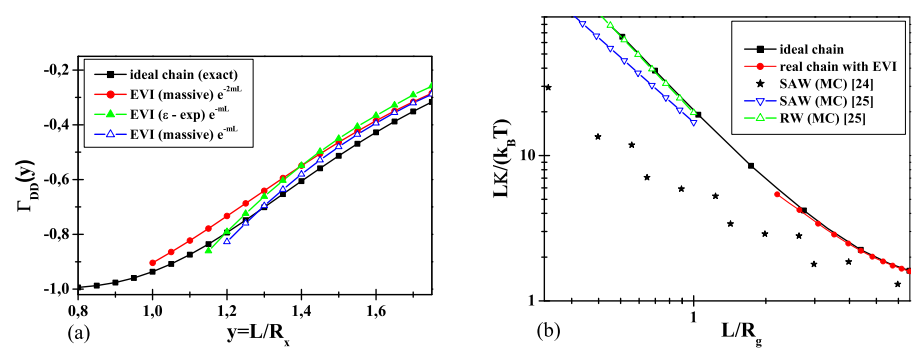

Fig. 4. (a) The dimensionless scaling functions of $\Gamma_{D D}(y)$ for linear polymer in a slit geometry of two repulsive walls (see[25]) in comparison to the results obtained in [24]. (b) Comparison of the analytical results obtained in [25] for the reduced canonical force and the results obtained by $\mathrm{MC}$ simulations for a trapped polymer between two repulsive walls.

\section{Conclusions and discussions}

In [25] we performed comparison of the obtained in the framework of the massive field theory in fixed space dimensions $d=3$ results for the scaling functions of the depletion interaction potential and the depletion force for the case of two repulsive walls and the results obtained using a dimensionally regularized continuum version of the field theory with minimal subtraction of poles in $\epsilon=4-d$ expansion. As it is possible to see from Figure 4a, the results obtained within the framework of both analytical methods are in good quantitative agrement. The obtained analytical results indicate that the reduction in the depletion effect due to the EVI is weaker within the massive field theory approach as compared to the results obtained using the $\epsilon$ - expansion. Besides, in [25] we obtained very good agreement of our analytical results for the reduced canonical force $L K / k_{B} T$ and the results obtained by Hsu and Grasberger [28] based on the lattice $\mathrm{MC}$ algorithm on a regular cubic lattice in three dimensions, as it is possible to see on Figure 4b. In this respect we should mention that in general case the canonical free energy can be obtained via the Legendre transform from the grand canonical one in the thermodynamic limit $(\tilde{N}, V \rightarrow \infty)$ (for details see [25]). In a series of papers $[24,25]$ the good qualitative agreement between analytical results and the experimental results [8] for the depletion interaction potential between the spherical colloidal particle of big size immersed in the dilute solution of nonionic linear polymer chains and the wall of the container were obtained.

The present investigations show that the scaling functions for the depletion interaction potential and the depletion force between two repulsive, two inert walls or two mixed walls induced by the dilute solution of phantom ideal ring polymers and ring polymers with the EVI (see [56-58]) are characterized by completely different behavior than in the case of the dilute solution of linear polymers immersed in the slit geometry of two parallel walls (see [25]). We would like to recollect that in the case of linear ideal polymers in confined geometry of two inert walls in [25] we obtained that the scaling functions for the depletion interaction potential and the depletion force were equal to zero. The obtained in [56-58] results indicate that ring polymers due to the complexity of chain topology and from entropical reasons prefer to escape from 


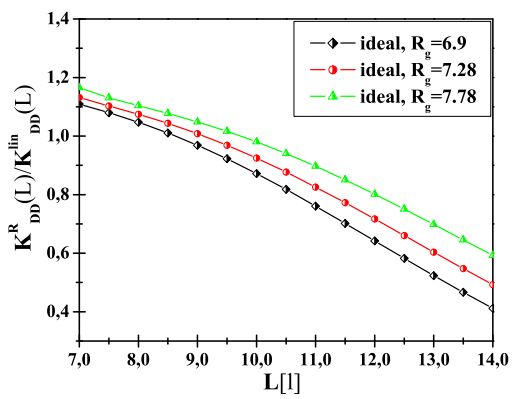

Fig. 5. The ratio $K_{D D}^{R}(L) / K_{D D}^{l i n}(L)$ of the force for phantom ideal ring polymers and the force for ideal linear polymers in the slit geometry of two parallel repulsive walls as function of the distance $L$ (in $l$ units) between the walls for different values of $R_{g}$ (see $[56,57]$ ).

the space not only between two repulsive walls but also in the case of two inert walls. As a result, the attractive depletion force arises which decreases as the width of slit increases. Besides, the depletion force between one repulsive and the other one inert wall induced by the dilute solution of ideal ring polymers becomes repulsive in the region $y \geq 1$. The similar behaviour was observed for binary liquid mixture confined in the slit geometry of two parallel walls with mixed boundary conditions as it was shown in [72]. Such behaviour of the dilute solution of phantom ideal ring polymers in confined geometries of two parallel walls with mixed boundary conditions can be used for the production of new types of micro- and nano- electromechanical devices, because it allows to reduce the static friction in such systems.

In order to compare our results with the results obtained in the framework of other methods we performed calculations of the ratio $K_{D P}^{R}(L) / K_{D D}^{l i n}(L)$ of the entropically induced force for phantom ideal ring polymers $K_{D D}^{R}$ and the corresponding force for ideal linear polymers $K_{D D}^{l i n}$ in the slit geometry of two parallel repulsive walls as function of the distance $L$ (in $l$ units) between the walls for different values of the radius of gyration $R_{g}$ (see Fig. 5). The calculations presented in Figure 5 were performed for different values of radius of gyrations [37]: $R_{g}\left(12_{1}\right)=6.9 \pm 0.01[l]$, $R_{g}\left(9_{1}\right)=7.28 \pm 0.01[l]$, and $R_{g}\left(6_{1}\right)=7.78 \pm 0.01[l]$ which correspond to the ring polymers with different knot types: $12_{1} ; 9_{1} ; 6_{1}$, where $C_{p-}$ is a standard notation [73], where $C$ denotes the minimum number of crossings in any projection on a plane and $p$ - is used in order to distinguish knot types with the same $C$. We observed that in the wide slit region ring polymers with less complex knot types (with bigger radius of gyration) in a ring topology exert higher forces on the confining repulsive walls at the same length $L$. As it is possible to see from Figure 5 our results for the ratio of the resulting entropic forces $K_{D D}^{R}(L) / K_{D D}^{l i n}(L)$ are in good qualitative agreement with the previous results obtained by Matthews et al. (see Fig. 2 in [37]) in the framework of bead-spring model. The difference between our results and the results obtained by Matthews et al. arises due to the fact that in [37] calculations were performed for relatively short polymers with polymer length of order $N \sim 300$ units for different values of the radius of gyration.

Taking into account the Derjaguin approximation [71] we obtained the results for the depletion interaction potential and the depletion force for the dilute solution of ring polymers immersed in the solution of mesoscopic spherical colloidal particles. We performed investigation of the depletion effect for mesoscopic colloidal particles with different radii and different adsorbing or repelling properties with respect to ring polymers in the solution. As it is possible to see from the obtained in equation (11) and Table 2 results the absolute value of the depletion force between the spherical colloidal particle and the wall is bigger than for the case of two spherical colloidal particles. 
This feature is universal and does not depend on the boundary condition type. In the case when two colloidal particles have the same radius, the corresponding depletion force is twice smaller than for the case of the particle near the wall. The further theoretical and experimental investigations of the dilute and semidilute solutions of linear and ring polymers with the EVI immersed in the slit geometry of two inert walls or mixed walls is the task of great interest which is under consideration.

This work in part was supported by grant $P-22 / 29.07 .2014$ under a bilateral agreement between the PAS and the BAS (Z.U. and H.Ch.).

\section{References}

1. L.A. Lyon, A. Fernandez-Nieves, Ann. Rev. Phys. Chem. 63, 25 (2012)

2. M. Fuchs, K.S. Schweizer, J. Phys. Condensed Matter 14, R239 (2002)

3. X. Chen, J. Cai, H. Liu, Y. Hu, Mol. Simul. 32, 877 (2006)

4. T. Cosgrove, Colloid Science Principles, Methods and Applications (Wiley, 2010)

5. N.W. Lekkerkerker Henk, R. Tuinier, Colloids and the Depletion Interaction, Lecture Notes in Physics 833 (Springer, Netherlands, 2011)

6. D.H. Napper, Polymeric Stabilization of Colloidal Dispersions (Academic, New York, 1983)

7. B.L. Carvalho et al., Macromolecules 26, 4632 (1993)

8. D. Rudhardt, C. Bechinger, P. Leiderer, Phys. Rev. Lett. 81, 1330 (1998)

9. R. Verma, J.C. Crocker, T.C. Lubensky, A.G. Yodh, Phys. Rev. Lett. 81, 4004 (1998)

10. Y.N. Ohshima, H. Sakagami, K. Okumoto, A. Tokoyoda, T. Igarashi, K.B. Shintaku, S. Toride, H. Sekino, K. Kabuto, I. Nishio, Phys. Rev. Lett. 78, 3963 (1997)

11. R.A. Curtis, L. Lue, Curr. Opin. Colloid Interface Sci. 20, 19 (2015)

12. S. Asakura, F. Oosawa, J. Chem. Phys. 22, 1255 (1954)

13. S. Asakura, F. Oosawa, J. Polym. Sci. 33, 183 (1958)

14. J.F. Joanny, L. Leibler, P.G. de Gennes, J. Polym. Sci. 17, 1073 (1979)

15. P.G. de Gennes, Phys. Lett. A 38, 339 (1972)

16. P.G. de Gennes, Scaling Concepts in Polymer Physics (Cornell University Press, New York, 1979)

17. T. Odijk, Macromolecules 29, 1842 (1996)

18. T. Odijk, J. Chem. Phys. 106, 3402 (1996)

19. J.F. Joanny, J. Phys. (France) 49, 1981 (1988)

20. R. Lipowsky, Europhys. Lett. 30, 197 (1995)

21. C.E. Cordeiro, J. Phys. Chem. Solids 60, 1645 (1999)

22. Y. Singh, S. Kumar, D. Giri, J. Phys. A 32, L407 (1999)

23. E. Eisenriegler, Phys. Rev. E 55, 3116 (1997)

24. F. Schlesener, A. Hanke, R. Klimpel, S. Dietrich, Phys. Rev. E 63, 041803 (2001)

25. D. Romeis, Z. Usatenko, Phys. Rev. E 80, 041802 (2009)

26. A. Milchev, K. Binder, Eur. Phys. J. B 3, 477 (1998)

27. A. Milchev, K. Binder, Eur. Phys. J. B 13, 607 (2000)

28. H.P. Hsu, P. Grasberger, J. Chem. Phys. 120, 2034 (2004)

29. Z. Usatenko, J. Chem. Phys. 134, 024119 (2011)

30. Z. Usatenko, J. Mol. Liq. 164, 59 (2011)

31. A. Bringer, E. Eisenriegler, F. Schlesener, A. Hanke, Eur. Phys. J. B 11, 101 (1999)

32. A. Hanke, E. Eisenriegler, S. Dietrich, Phys. Rev. E 59, 6853 (1999)

33. J. Marek et al., Cytometry Part A 63A, 87 (2005)

34. G. Witz, K. Rechendorff, J. Adamcik, G. Dietler, Phys. Rev. Lett. 106, 248301 (2011)

35. J. Berg, J. Tymoczko L. Stryer, Biochemistry, 5th edn. (New Yok, W.H. Freeman and Co., 2002)

36. J. Arsuaga, M. Vazquez, S. Trigueros, D.W. Sumners, J. Roca, Proc. Natl. Acad. Sci. U.S.A. 99, $5373(2002)$ 
37. R. Matthews, A.A. Louis, J.M. Yeomans, Mol. Phys. 109, 1289 (2011)

38. R.V. Miller, Sci. Am. 278, 66 (1998)

39. S. Jun, B. Mulder, Proc. Natl. Acad. Sci. U.S.A. 103, 12388 (2006)

40. C.O. Dietrich-Buchecker, J.P. Sauvage, Angew. Chem. Int. Edn. Engl. 28, 189 (1989)

41. A. Dobay et al., Proc. Natl. Acad. Sci. U.S.A. 100, 5611 (2003)

42. E. Ercolini et al., Phys. Rev. Lett. 98, 058102 (2007)

43. K. Alim, E. Frey, Phys. Rev. Lett. 99, 198102 (2007)

44. E.J. Rawdon et al., Macromolecules 41, 8281 (2008)

45. E.J. Janse van Rensburg, S.G. Whittington, J. Phys. A: Math. Gen. 23, 3573 (1990)

46. S. Quake, Phys. Rev. Lett. 73, 3317 (1994)

47. E.J. Janse van Rensburg, J. Stat. Mech. P03001 (2007)

48. E.J. Janse van Rensburg, E. Orlandini, M.C. Tesi, S.G. Whittington, J. Phys. A: Math. Theor. 41, 015003 (2008)

49. D. Gasumova, E.J. Janse van Rensburg, A. Rechnitzer, J. Stat. Mech. P09004 (2012)

50. E.J. Janse van Rensburg, A. Rechnitzer, J. Phys. A: Math. Theor. 42, 335001 (2009)

51. C. Micheletti, E. Orlandini, Macromolecules 45, 2113 (2012)

52. B. Li, Z.Y. Sun, L.J. An, Z.G. Wang, Macromolecules 48, 8675 (2015)

53. D. Reith, P. Cifra, A. Stasiak, P. Virnau, Nucleic Acids Res. 40, 5129 (2012)

54. B. Trefz, J. Siebert, P. Virnau, Proc. Natl. Acad. Sci. USA 111, 7948 (2014)

55. M. Marenz, W. Janke, Phys. Rev. Lett. 116, 128301 (2016)

56. Z. Usatenko, J. Halun, P. Kuterba, Condens. Matter Phys. 19, 43602 (2016)

57. Z. Usatenko, J. Halun, J. Stat. Mech. AA5285 (2016)

58. Z. Usatenko, P. Kuterba, H. Chamati, J. Halun, J. Phys. Conf. Ser. (2016)

59. H.W. Diehl, M. Shpot, Nucl. Phys. B 528, 595 (1998)

60. J. Zierenberg, M. Mueller, P. Schierz, M. Marenz, W. Janke, J. Chem. Phys. 141, 114908 (2016)

61. J. des Cloizeaux, G. Jannink, Polymers in Solution (Oxford, Clarendon Press, 1990)

62. E. Eisenriegler, Polymers Near Surfaces (World Scientific Publishing, Singapore, 1993)

63. E. Orlandini, S. Whittington, Rev. Mod. Phys. 79, 611 (2007)

64. K. Binder, in Phase Transitions and Critical Phenomena, edited by C. Domb, J.L. Lebowitz, vol. 8 (Academic Press, London, 1983)

65. H.W. Diehl, in Phase Transitions and Critical Phenomena, edited by C. Domb, J.L. Lebowitz, vol. 10 (Academic Press, London 1986)

66. M. Bachmann, W. Janke, Phys. Rev. Lett. 95, 058102 (2005)

67. M. Möddel, M. Bachmann, W. Janke, J. Phys. Chem. B 113, 3314 (2009)

68. M. Möddel, W. Janke, M. Bachmann, Phys. Chem. Chem. Phys. 12, 11548 (2010)

69. L. Schäfer, Excluded Volume Effects in Polymer Solutions as Explained by the Renormalization Group (Springer, Heidelberg, 1998)

70. G. Parisi, Statistical Field Theory (Addison-Wesley, Redwood City, 1988)

71. B.V. Derjaguin, Kolloid-Z. 69, 155 (1934)

72. C. Hertlein, L. Helden, A. Gambassi, S. Dietrich, C. Bechinger, Nature 451, 172 (2008)

73. E. Orlandini, S. Whittington, Rev. Mod. Phys. 79, 611 (2007)

Open Access This is an Open Access article distributed under the terms of the Creative Commons Attribution License (http://creativecommons.org/licenses/by/4.0), which permits unrestricted use, distribution, and reproduction in any medium, provided the original work is properly cited. 